\title{
An approach to a patient with infective endocarditis
}

\author{
J Hitzeroth, ${ }^{1}$ MB ChB, DA (SA), FCP (SA), Cert Cardiology (SA); N Beckett, ${ }^{2}$ MB ChB, PG Dip FM (SA); \\ P Ntuli, ${ }^{3} \mathrm{MB} \mathrm{ChB}, \mathrm{FCP}(\mathrm{SA})$, Cert Cardiology (SA) \\ ${ }^{1}$ Private Practice, Vincent Pallotti Hospital, Cape Town, South Africa \\ ${ }^{2}$ Division of Family Medicine, School of Public Health and Family Medicine, Faculty of Health Sciences, University of Cape Town, South Africa \\ ${ }^{3}$ Division of Cardiology, Department of Medicine, Faculty of Health Sciences, University of Cape Town, and Groote Schuur Hospital, Cape Town, \\ South Africa
}

Corresponding author: J Hitzeroth (jens314@gmail.com)

\begin{abstract}
Although infective endocarditis (IE) is relatively uncommon, it remains an important clinical entity with a high in-hospital and 1-year mortality. It is most commonly caused by viridans streptococci. Staphylococcus aureus is responsible for a malignant course of IE and often requires early surgery to eradicate. Other rarer causes are various bacilli, including the HACEK (Haemophilus, Actinobacillus, Cardiobacterium, Eikenella and Kingella spp.) group of organisms and fungi. The clinical presentation varies. Patients may present with a nonspecific illness, valve dysfunction, heart failure (HF) and symptoms due to peripheral embolisation. The diagnosis is traditionally based on the modified Duke criteria and rests mainly on clinical features and to a lesser extent on certain laboratory findings, microbiological assessment and cardiovascular imaging. Identification of the offending micro-organism is not only important from a diagnostic point of view, but also makes targeted antibiotic treatment possible and provides useful prognostic information. A significant proportion of microbiological cultures are negative, frequently owing to the administration of antibiotics prior to appropriate culture. Blood-culture-negative IE poses significant diagnostic and treatment challenges. The course of the disease is frequently complicated, and sequelae include HF, local intracardiac extension of infection (abscess, fistula, pseudoaneurysm), stroke and intracranial haemorrhage due to septic emboli or mycotic aneurysm formation as well as renal injury. Management includes prolonged intravenous antibiotics and consideration for early surgery with removal of infective tissue and valve replacement in patients who have poor prognostic features or complications. Antibiotic administration for at-risk patients to prevent bacteraemia during specific procedures (particularly dental) is recommended to prevent IE. The patient population who would benefit from antibiotic prophylaxis has become increasingly restricted, and guidelines recommend prophylaxis only for patients with cyanotic congenital heart disease, prosthetic heart valves and a previous episode of IE. The management of a patient with IE is challenging and often requires multidisciplinary input from an IE heart team, which includes cardiologists.
\end{abstract}

S Afr Med J 2016;106(2):145-150. DOI:10.7196/SAMJ.2016.v106i2.10327

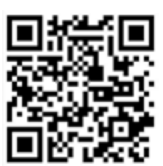

Infective endocarditis (IE) remains a challenging clinical entity. ${ }^{[1]}$ It has a relatively low incidence, the clinical presentation varies widely and the offending organisms cover a large spectrum of microbiological species. It is therefore not uncommon that the diagnosis is delayed.

Despite advances in its management, it remains a condition with a high mortality and significant morbidity. Paucity of data, particularly in Africa where rheumatic valvular heart disease is still highly prevalent, makes decisions regarding the optimal treatment of this disease very difficult. To complicate matters, there has been considerable controversy surrounding prevention of the disease.

The objective of this article is to inform the clinician on the current state of knowledge of IE in order to provide a practical approach to the patient with suspected IE.

\section{Bacteriology}

Viridans streptococci account for $50-70 \%$ of native valve IE. Staphylococcus aureus is responsible for a further $25 \%$. Various bacilli, the HACEK group of organisms (Haemophilus, Actinobacillus, Cardiobacterium, Eikenella and Kingella spp.) and fungi are rare.

The offending organisms in prosthetic valve endocarditis are frequently Staphylococcus epidermidis or S. aureus.

\section{Prevention}

Previously, antibiotic prophylaxis was administered widely to patients with various predisposing cardiac conditions. In 2002 the indications for antibiotic prophylaxis were restricted to a much smaller population of patients with cardiac pathology, ${ }^{[2]}$ for various reasons:

- Bacteraemia occurs more frequently during routine activities (e.g. toothbrushing) and the risk of endocarditis may be related to generally poor dental health rather than occasional high-grade bacteraemia following a dental procedure.

- There is poor correlation between invasive dental procedures and IE

- The estimated risk of IE following a dental procedure is very low.

- Antibiotic prophylaxis carries a small but definite risk of anaphylaxis/severe allergic reaction.

- Prophylactic antibiotic use may contribute to increased resistant bacteria.

- There are no randomised controlled trials that have investigated whether antibiotic prophylaxis does in fact reduce the incidence of IE.

In a recent analysis in the UK, the incidence of IE increased significantly after introduction of the restricted antibiotic prophylaxis guidelines. It is not entirely certain what conclusions can be drawn from this observational data, where confounding factors cannot be excluded. The finding has not been confirmed in various observational studies in other countries.

The current European Society of Cardiology (ESC) guidelines still recommend that antibiotic prophylaxis should be considered for:

- Patients with a prosthetic valve or where prosthetic material was used for surgical repair of a valve. 
- Patients with a previous episode of IE.

- Any type of cyanotic congenital heart disease.

- Any coronary heart disease repaired with prosthetic material up to 6 months after the procedure or lifelong if there is residual shunt/ valvular regurgitation. ${ }^{[3]}$

Furthermore, the use of antibiotic prophylaxis is limited to dental procedures requiring manipulation of the gingival/periapical tooth region or perforation of the oral mucosa. Antibiotic prophylaxis is not recommended for less-invasive procedures such as bronchoscopy, gastroscopy, colonoscopy, cystoscopy or skin/soft-tissue procedures. This also includes caesarean sections and vaginal delivery. Respiratory, gastrointestinal tract or genitourinary procedures where antibiotic prophylaxis is given to prevent wound infection or sepsis should receive the appropriate antibiotic.

The recommended regimen for patients in whom antibiotic prophylaxis is indicated is amoxicillin $2 \mathrm{~g}$ orally or intravenously (IV) 30 - 60 minutes prior to the procedure. Patients with a penicillin allergy should receive clindamycin $600 \mathrm{mg}$ orally or IV.

Lastly, the following general measures to reduce bacteraemia are felt to be very important:

- Strict dental and cutaneous hygiene.

- Disinfection of any wounds.

- Eradication of chronic bacterial carriage: skin/nasal/urine.

- Aggressive therapy of any bacterial infection.

- Strict infection control measures for any procedure.

There have been case reports of IE after body piercing or tattooing; therefore, these should be strongly discouraged in at-risk individuals.

A final comment must be made surrounding so-called healthcareassociated IE. This accounts for up to $30 \%$ of all cases of IE. To prevent this, the following points are important:

- The often unnecessary use of IV catheters should be limited.

- A strict aseptic technique should be followed, particularly when placing central catheters.

- Catheters should be changed routinely every 3 - 4 days.

\section{Table 1. Definition of IE according to modified Duke criteria}

\begin{tabular}{l} 
Definite IE \\
\hline Pathological criteria \\
Micro-organism demonstrated by culture or histological \\
examination of a vegetation, a vegetation that has embolised, \\
or an intracardiac abscess specimen, or \\
Pathological lesions vegetation or intracardiac abscess confirmed \\
by histological examination showing active endocarditis \\
Clinical criteria \\
2 major criteria, or \\
1 major criterion and 3 minor criteria, or \\
5 minor criteria \\
Possible IE
\end{tabular}

1 major criterion and 1 minor criterion, or

3 minor criteria

\section{Rejected IE}

Firm alternative diagnosis, or

Resolution of symptoms suggesting IE after antibiotic therapy for $<4$ days, or

No pathological evidence of IE at surgery or autopsy, with antibiotic therapy for $<4$ days, or

Does not meet criteria for possible IE, as above

\section{Diagnosis}

The modified Duke criteria provide a formal framework for the diagnosis of IE and are shown in Tables 1 and $2 .{ }^{[4]}$ An important caveat is that the Duke criteria have a sensitivity of $80 \%$ for the diagnosis of IE. ${ }^{[5]}$ It is therefore possible to miss IE if one applies the Duke criteria too strictly.

The diagnosis is based on four distinct legs:

- Clinical features.

- Laboratory findings.

- Imaging.

- Microbiological tests.

\section{Table 2. Definition of terms used in the Duke criteria}

\begin{tabular}{l} 
Major criteria \\
\hline Blood culture positive for IE \\
Typical micro-organism consistent with IE from two separate \\
blood cultures \\
Viridians streptococci, Streptococcus gallolyticus, HACEK \\
group, S. aureus, or \\
Community-acquired enterococci, in the absence of a primary \\
focus, or
\end{tabular}

Micro-organisms consistent with IE from persistently positive blood cultures defined as follows:

$>2$ positive blood cultures of blood samples drawn $>12$ hours apart, or

All of 3 or a majority of $>4$ separate blood cultures (with first and last sample drawn $>1$ hour apart), or

Single positive blood culture for Coxiella burnetti or IgG titre $>1: 800$

Imaging positive for IE

Echocardiogram positive for IE

Vegetation, or

Abscess, pseudoaneurysm, intracardiac fistula, or

Valvular perforation or aneurysm, or

New partial dehiscence of prosthetic valve

Definite paravalvular lesions on cardiac CT

Abnormal activity around the site of prosthetic valve implantation detected by ${ }^{18} \mathrm{~F}-\mathrm{FDG}$ PET/CT (only if prosthesis was implanted for $>3$ months) or radio-labelled leukocytes SPECT

\section{Minor criteria}

Predisposition, such as predisposing heart condition or IV drug use Fever defined as temperature $>38^{\circ} \mathrm{C}$

Vascular phenomena (including those detected by imaging only)

Major arterial emboli

Septic pulmonary infarcts

Infectious aneurysm

Intracranial haemorrhage

Conjunctival haemorrhages

Janeway's lesions

Immunological phenomena

Glomerulonephritis

Osler's nodes

Roth's spots

Rheumatoid factor

Microbiological evidence

Positive blood culture but does not meet major criteria for

blood cultures as noted above, or

Serological evidence of active infection with organism consistent with IE

${ }^{18} \mathrm{~F}$-FDG PET/CT = fluorine- 18 fluorodeoxyglucose positron emission tomography/ computed tomography; SPECT = single-photon emission computed tomography. 


\section{Clinical features}

IE usually has an acute onset, and it often causes multisystem disease with skin manifestations that can imitate a number of diseases ${ }^{[6]}$ In a primary care setting it is important to have a high index of suspicion, and the clinical history of a patient with unexplained high fever should always include asking about possible risk factors such as previous cardiac valvular disease, IV drug use, the presence of prosthetic devices (prosthetic valves, orthopaedic hardware), as well as cardiac implantable electronic devices such as pacemakers, which can be possible sources of infection.

The clinical picture of IE varies from nonspecific symptoms to symptoms suggestive of pre-existing cardiac valvular disease, as well as embolic phenomena. Nonspecific symptoms include a high fever (in about 80 - $90 \%$ of cases), headache, anorexia, myalgia, night sweats and joint pains. ${ }^{[7,8]}$ The patient often presents acutely, with no prior history of valvular disease or existing cardiac murmurs.

Patients with pre-existing valve lesions and congenital heart disease usually present with the subacute form of the disease. There is an overlap of symptoms with those found in acute cases, because the clinical picture is influenced by the source or site of the infection, as well as the organism involved. About $99 \%$ of these patients will have a cardiac murmur but only $10 \%$ of these patients have a change in the characteristics of the murmur. ${ }^{[9]}$ Patients who have leftsided valvular insufficiency may present with an acute onset of congestive cardiac failure. Splenomegaly is found in about $11 \%$ of patients with long-standing disease, but clubbing of fingers and toes is rare.

The classic signs of IE are summarised in Table 3.

Patients who have embolic complications can present with neurological outcomes ( $40 \%$ of cases), which include hemiparesis, paralysis and aphasia. In $50 \%$ of patients who present with cerebral emboli as the first manifestation of IE, there is a 2 4 times increased mortality rate. It is therefore important to enquire about the previously mentioned risk factors as possible sources of infection, especially in a young person who presents with stroke symptoms. Other manifestations include glomerulonephritis and renal failure. Systemic septic emboli (S. aureus infection) can result in abscess formation in almost any organ, including the heart, brain and kidneys.

IV drug users (IVDUs) are susceptible to tricuspid IE. These patients have pulmonary symptoms, in addition to symptoms such as high fever and petechiae. IVDUs with concurrent HIV infection are at increased risk of tricuspid valve endocarditis. Patients with advanced stages of HIV disease have a greater mortality risk of IE. ${ }^{[10]}$

\section{Laboratory findings}

None of the various laboratory investigations that are performed are diagnostic, and generally have poor positive predictive values. The tests are at most supportive in the diagnosis of IE. A raised white cell count or other marker of sepsis such as a raised erythrocyte sedimentation rate, C-reactive protein or procalcitonin may be found. Anaemia of chronic disease can be present. Patients may have low complement levels or elevated rheumatoid factor. An elevated creatinine may be due to acute tubular necrosis, immune-complex mediated nephritis or renal emboli.

\section{Imaging}

\section{Echocardiography}

Echocardiography remains one of the most useful investigations in the evaluation of someone with suspected IE. ${ }^{[11]}$ If the transthoracic echocardiogram is negative, one should proceed to transoesophageal echocardiography to obtain the necessary image quality to look for small vegetations or early intracardiac complications (e.g. perivalvular abscesses). ${ }^{[12]}$ Transoesophageal echocardiography must also be performed in patients with suspected prosthetic valve endocarditis. The resolution of a transthoracic echocardiogram is not adequate in this setting. Patients with $S$. aureus bacteraemia often have underlying IE with a very poor prognosis, and an echocardiogram is recommended in this setting to look for IE. ${ }^{[13]}$ It needs to be emphasised that a normal echocardiogram does not rule out IE and if the clinical suspicion remains high, the study should be repeated 5 days later.

\section{Computed tomography scanning}

Multislice computed tomography (CT) may be helpful to detect the following:

- CT coronary angiography is helpful in patients in whom a preoperative angiogram is indicated. The risks of invasive angiography including potential dislodgement of vegetation are thus avoided.

- Multislice CT of the lungs, spleen or brain can detect embolic lesions, which can help to confirm the diagnosis of IE.

- Cardiac CT can detect intracardiac/perivalvular abscesses and fistulae.

\section{Magnetic resonance imaging}

This may help in detecting cerebral lesions. Cardiovascular magnetic resonance may be helpful in identifying vegetations, myocardial abscesses and other associated complications.
Table 3. Clinical signs of IE

\begin{tabular}{|c|c|c|}
\hline Clinical sign & Occurrence, \% & Description \\
\hline Petechiae & $\begin{array}{l}\text { Common, } \\
\text { nonspecific }\end{array}$ & $\begin{array}{l}\text { Common, nonspecific and found on } \\
\text { extremities, mucosa and palate }\end{array}$ \\
\hline Splinter haemorrhages & 8 & Dark, linear lesions in nail beds \\
\hline Osler's nodes & & $\begin{array}{l}\text { Tender, papulopustules on distal pads of } \\
\text { digits }\end{array}$ \\
\hline Roth's spots & 5 & $\begin{array}{l}\text { Retinal haemorrhages with small, clear } \\
\text { centres }\end{array}$ \\
\hline Janeway's lesions & 5 & $\begin{array}{l}\text { Painless erythematous macules on palms of } \\
\text { hands and soles of feet }\end{array}$ \\
\hline Conjunctival haemorrhages & 5 & Bright red/dark red patch on the sclera \\
\hline Haematuria & 25 & Blood in the urine \\
\hline
\end{tabular}

Table 4. Empirical therapy of IE

\begin{tabular}{llll}
\hline Condition & Antibiotics & Dose & Duration (weeks) \\
\hline Native valve IE & Penicillin & $6 \mathrm{mU}$ given 6-hourly IV & 4 \\
& Gentamicin & $3 \mathrm{mg} / \mathrm{kg} /$ day given 12-hourly IV & 2 \\
Prosthetic valve IE & Vancomycin & $30 \mathrm{mg} / \mathrm{kg} /$ day given 12-hourly IV & 6 \\
& Rifampicin & $15 \mathrm{mg} / \mathrm{kg} /$ day given 12-hourly po & 6 \\
& Gentamicin & $3 \mathrm{mg} / \mathrm{kg} /$ day given 12-hourly IV & 2
\end{tabular}




\section{Nuclear imaging}

In centres with the necessary expertise and equipment, fluorine-18 fluorodeoxyglucose positron emission tomography/CT $\left({ }^{18} \mathrm{~F}-\mathrm{FDG}\right.$ $\mathrm{PET} / \mathrm{CT}$ ) can diagnose septic foci.

\section{Microbiological tests}

Blood cultures are critical in the diagnosis of IE. It is recommended that three sets are taken at least 30 minutes apart. Peripheral samples are preferred. Blood cultures do not need to be timed with fever spikes, as bacteraemia in IE is very constant. Similarly, a single positive blood culture should be interpreted carefully, as usually in IE all blood cultures will be positive. Positive blood cultures allow for identification of the causative organism as well as antibiotic susceptibility testing.

Unfortunately, in about one-third of cases blood cultures are negative. This frequently results in diagnostic dilemmas and considerable challenge in terms of therapy. By far the most common cause is prior antibiotic administration without appropriate blood cultures being taken at the time. In these cases it may be useful (if the clinical status of the patient allows) to stop the antibiotics and repeat blood cultures.

Another cause of blood-culture-negative IE is unusual organisms such as fungi and certain intracellular bacteria, which require specific growth media. Serology and polymerase chain reaction (PCR) for specific organisms (Coxiella, Bartonella and Brucella) may be helpful in these cases. ${ }^{[14]}$

Lastly, microbiological examination of resected tissue during surgery can be extremely helpful. All tissue excised during surgery should be sent for appropriate microbiological and histological investigation.

\section{Complications}

Not all the potential complications of IE are discussed here, but some of the more commonly occurring problems deserve special mention. Apart from heart failure, IE can manifest complications affecting the central nervous system, spleen, kidneys as well as blood vessels with the formation of mycotic aneurysms.

\section{Neurological}

About $20 \%$ of patients develop symptomatic neurological complications. These are usually due to septic emboli and may be the first manifestation of IE. The usual clinical presentation is with focal neurological signs due to an ischaemic stroke but more severe complications such as haemorrhage, meningitis and abscess formation can occur. Cardiac surgery to prevent further embolism should be considered early in patients with neurological complications.
Spleen

Splenic abscesses or rupture present with abdominal pain and often a failure of antibiotic therapy. Although this complication is rare, it is important to recognise as these patients may require splenectomy to cure their infection; however, small abscesses may still respond to antibiotics alone.

\section{Heart block}

This is a rare complication but usually indicates perivalvular spread of the infection and is therefore often an indication for surgery.

\section{Kidney}

Up to $30 \%$ of patients can develop acute renal failure. The following aetiologies should be considered:

- Acute tubular necrosis due to sepsis or haemodynamic compromise.

- Immune complex-mediated glomerulonephritis.

- Renal infarction due to emboli.

- Drug toxicity or drug-induced nephritis.

\section{Treatment}

The therapy of IE comprises appropriate antibiotic therapy, cardiac surgery to resect infected cardiac tissue (usually with valve replacement) and the treatment of complications.

\section{Antibiotic therapy}

Specific antibiotic therapy should be guided by the identification of the organism and results of antibiotic susceptibility testing. Tables 4 - 9 outline the empirical treatment of patients with IE (before blood culture results are available) as well as the antibiotic regimen for the most common organisms once they have been identified by cultures. ${ }^{[15]}$ It is strongly recommended to discuss the specific treatment regimen with a microbiologist/ infectious disease specialist.

\section{Surgery}

Surgery is indicated broadly for three reasons: the development of heart failure, inadequate response to antibiotic therapy and a reduction in the risk of septic embolism.

\section{- Heart failure}

Heart failure is usually on the basis of severe mitral or aortic regurgitation. Patients who do not respond to medical heart failure therapy should undergo surgery within 24 hours.
Table 5. Therapy for streptococcal IE (native valve)

\begin{tabular}{|c|c|c|c|}
\hline Condition & Antibiotics & Dose & Duration (weeks) \\
\hline $\begin{array}{l}\text { Viridans streptococci } \\
\text { susceptible to penicillin }\end{array}$ & Penicillin & $6 \mathrm{mU}$ given 6-hourly IV & 4 \\
\hline \multirow{2}{*}{$\begin{array}{l}\text { Viridans streptococci moderately } \\
\text { susceptible to penicillin }\end{array}$} & Penicillin & $6 \mathrm{mU}$ given 6 -hourly IV & 4 \\
\hline & Gentamicin & $\begin{array}{l}3 \mathrm{mg} / \mathrm{kg} / \text { day given } \\
\text { 12-hourly IV }\end{array}$ & 2 \\
\hline \multirow{2}{*}{$\begin{array}{l}\text { Viridans streptococci } \\
\text { moderately resistant to } \\
\text { penicillin }\end{array}$} & Penicillin & $6 \mathrm{mU}$ given 6-hourly IV & 4 \\
\hline & Gentamicin & $\begin{array}{l}3 \mathrm{mg} / \mathrm{kg} / \text { day given } \\
\text { 12-hourly IV }\end{array}$ & 4 \\
\hline \multirow{2}{*}{$\begin{array}{l}\text { Enterococci susceptible to } \\
\text { penicillin }\end{array}$} & Penicillin & $6 \mathrm{mU}$ given 6-hourly IV & 4 \\
\hline & Gentamicin & $\begin{array}{l}3 \mathrm{mg} / \mathrm{kg} / \text { day given } \\
\text { 12-hourly IV }\end{array}$ & 4 \\
\hline \multirow[t]{2}{*}{ Abiotrophia/Granulicatella } & Penicillin & $6 \mathrm{mU}$ given 6 -hourly IV & 4 \\
\hline & Gentamicin & $\begin{array}{l}3 \mathrm{mg} / \mathrm{kg} / \text { day given } \\
\text { 12-hourly IV }\end{array}$ & 4 \\
\hline \multirow[t]{2}{*}{$\begin{array}{l}\text { Viridans streptococci fully } \\
\text { resistant to penicillin }\end{array}$} & Vancomycin & $\begin{array}{l}30 \mathrm{mg} / \mathrm{kg} / \text { day given } \\
\text { 12-hourly IV }\end{array}$ & 6 \\
\hline & Gentamicin & $\begin{array}{l}3 \mathrm{mg} / \mathrm{kg} / \text { day given } \\
\text { 12-hourly IV }\end{array}$ & 6 \\
\hline \multirow[t]{2}{*}{$\begin{array}{l}\text { Enterococci resistant to } \\
\text { penicillin }\end{array}$} & Vancomycin & $\begin{array}{l}30 \mathrm{mg} / \mathrm{kg} / \text { day given } \\
\text { 12-hourly IV }\end{array}$ & 6 \\
\hline & Gentamicin & $\begin{array}{l}3 \mathrm{mg} / \mathrm{kg} / \text { day given } \\
\text { 12-hourly IV }\end{array}$ & 6 \\
\hline
\end{tabular}


Patients who respond to heart failure therapy should undergo surgery within 7 days. Patients who are left with valve lesions with no evidence of heart failure should be followed up and then operated on electively.

\section{- Inadequate response to antibiotic therapy}

This usually manifests as either persisting fever or persistently positive blood cultures for $>7$ days after initiation of appropriate antibiotic therapy. Another manifestation of uncontrolled infection is the development of local cardiac complications such as abscesses, false aneurysm, fistulae and vegetations that are enlarging. In these cases surgery within 7 days is advised to remove infective tissue and treat local complications. Some infections are caused by organisms (e.g. fungi or resistant organisms) that have a high likelihood of not being adequately treated with antibiotic therapy alone. In these patients, early surgery with removal of infective tissue should strongly be considered.

\section{- Reduction in embolism risk}

Patients who have vegetations $>10 \mathrm{~mm}$, in particular if they have had a previous embolic episode or other poor prognostic factors (e.g. heart failure), should be considered for early surgery. ${ }^{[16]}$

\section{Cardiac device-related IE}

Patients who develop IE with involvement of a pacemaker or ICD deserve a special mention because mortality in this setting is particularly high and treatment is often very difficult. Apart from following the principles outlined above to make the diagnosis, it needs to be emphasised that the treatment of IE in this setting should not involve prolonged antibiotic therapy only, but also complete removal of all device-associated hardware (pacemaker and leads). If this is not performed, recurrence frequently occurs.

\section{Prognosis}

Between $15 \%$ and $30 \%$ of patients will die from IE in hospital. Table 10 outlines the predictors of a poor outcome for these patients. Patients with heart failure, periannular complications or $S$. aureus infections are at highest risk of in-hospital death or need for surgery. The risk is cumulative and is $79 \%$ if a patient has all three of the aforementioned risk factors. ${ }^{[17,18]}$

\section{Conclusion}

IE is a serious condition with a high mortality. It is difficult to diagnose and treat, and optimal management often requires input from multiple medical as well as surgical disciplines. Early surgery is frequently beneficial in patients with IE, and access to a good cardiac surgery service is therefore of paramount importance. As a final point we recommend that all patients with suspected IE are referred to a specialist centre for investigation and management.

Table 6. Therapy for streptococcal IE (prosthetic valve)

\begin{tabular}{llll}
\hline Condition & Antibiotics & Dose & Duration (weeks) \\
\hline Viridans streptococci susceptible to penicillin & Penicillin & $6 \mathrm{mU}$ given 6-hourly IV & 6 \\
Viridans streptococci moderately susceptible to penicillin & Gentamicin & $3 \mathrm{mg} / \mathrm{kg} /$ day given 12-hourly IV & 2 \\
& Penicillin & $6 \mathrm{mU}$ given 6-hourly IV & 6 \\
Viridans streptococci moderately resistant to penicillin & Gentamicin & $3 \mathrm{mg} / \mathrm{kg} /$ day given 12-hourly IV & 6 \\
& Penicillin & $6 \mathrm{mU}$ given 6-hourly IV & 6 \\
Enterococci susceptible to penicillin & Gentamicin & $3 \mathrm{mg} / \mathrm{kg} /$ day given 12-hourly IV & 6 \\
& Penicillin & $6 \mathrm{mU}$ given 6-hourly IV & 6 \\
Abiotrophia/Granulicatella & Gentamicin & $3 \mathrm{mg} / \mathrm{kg} /$ day given 12-hourly IV & 6 \\
& Penicillin & $6 \mathrm{mU}$ given 6-hourly IV & 6 \\
Viridans streptococci fully resistant to penicillin & Gentamicin & $3 \mathrm{mg} / \mathrm{kg} /$ day given 12-hourly IV & 6 \\
& Vancomycin & $30 \mathrm{mg} / \mathrm{kg} /$ day given 12-hourly IV & 6 \\
Enterococci resistant to penicillin & Gentamicin & $3 \mathrm{mg} / \mathrm{kg} /$ day given 12-hourly IV & 6
\end{tabular}

Table 7. Therapy for staphylococcal IE

\begin{tabular}{|c|c|c|c|}
\hline Condition & Antibiotics & Dose & Duration (weeks) \\
\hline Native valve: cloxacillin/methicillin sensitive & Cloxacillin & 2 g given 6-hourly IV & $4-6$ \\
\hline \multirow[t]{2}{*}{ Native valve: cloxacillin/methicillin resistant } & Vancomycin & $30 \mathrm{mg} / \mathrm{kg} /$ day given 12 -hourly IV & $4-6$ \\
\hline & Rifampicin & $15 \mathrm{mg} / \mathrm{kg} /$ day given 12 -hourly po & $4-6$ \\
\hline \multirow[t]{3}{*}{ Prosthetic valve: cloxacillin/methicillin sensitive } & Cloxacillin & 2 g given 6-hourly IV & $6-8$ \\
\hline & Rifampicin & $15 \mathrm{mg} / \mathrm{kg} /$ day given 12 -hourly po & $6-8$ \\
\hline & Gentamicin & $3 \mathrm{mg} / \mathrm{kg} /$ day given 12 -hourly IV & 2 \\
\hline \multirow[t]{3}{*}{ Prosthetic valve: cloxacillin/methicillin resistant } & Vancomycin & $30 \mathrm{mg} / \mathrm{kg} /$ day given 12 -hourly IV & $6-8$ \\
\hline & Rifampicin & 15 mg/kg/day given 12-hourly po & $6-8$ \\
\hline & Gentamicin & $3 \mathrm{mg} / \mathrm{kg} /$ day given 12 -hourly IV & 2 \\
\hline
\end{tabular}


Table 8. Therapy for blood culture negative IE

\begin{tabular}{llll}
\hline Pathogen & Antibiotics & Dose & Duration (weeks) \\
\hline Brucella & Doxycycline & $200 \mathrm{mg}$ daily po & $>3$ \\
& Cotrimoxazole & $960 \mathrm{mg}$ 12-hourly po & \\
& Rifampicin & $300-600 \mathrm{mg}$ daily po & \\
Bartonella & Doxycycline & $200 \mathrm{mg}$ daily po & 6 \\
& Gentamicin & $1 \mathrm{mg} / \mathrm{kg}$ 8-hourly IV & \\
Coxiella burnetti (Q fever) & Doxycycline & $100 \mathrm{mg}$ 12-hourly po & $>18$ \\
& Ciprofloxacin & $250 \mathrm{mg}$ 12-hourly po &
\end{tabular}

Table 9. Therapy for HACEK group IE

\begin{tabular}{llll}
\hline Condition & Antibiotic & Dose & Duration (weeks) \\
\hline HACEK organisms & Ceftriaxone & 1 g daily IV/IM & 4
\end{tabular}

Table 10. Predictors of poor outcome in patients with $\mathrm{IE}^{[3]}$

Patient factors
Older age
Prosthetic valve IE
Diabetes mellitus
Comorbidity (e.g. frailty, immunosuppression, renal/pulmonary disease)
Complications
Heart failure
Renal failure
Greater than moderate area of ischaemic stroke
Brain haemorrhage
Septic shock
Micro-organism
S. aureus
Fungi
Non-HACEK Gram-negative bacilli
Echocardiographic findings
Periannular complications
Severe left-sided valve regurgitation
Low left-ventricular ejection fraction
Pulmonary hypertension
Large vegetations
Severe prosthetic valve dysfunction
Premature mitral valve closure and other signs of elevated diastolic pressures

\section{References}

1. Thuny F, Grisoli D, Collart F, Habib G, Raoult D. Management of infective endocarditis: Challenges and perspectives. Lancet 2012;379(9819):965-975. [http://dx.doi.org/10.1016/S01406736(11)60755-1]

2. Duval X, Leport C. Prophylaxis of infective endocarditis: Current tendencies, continuing controversies. Lancet Infect Current tendencies, continuing controversies. Lancet Infect
Dis 2008;8(4):225-232. [http://dx.doi.org/10.1016/S1473is $2099(08) 70064-1]$

3. Habib G, Lancellotti P, Antunes MJ. ESC guidelines for the management of infective endocarditis. Eur Heart J 2015;36:3075-3128. [http://dx.doi.org/10.1093/eurheartj/ ehv319]

4. Li J, Sexton DJ, Mick N, et al. Proposed modifications to the Duke criteria for the diagnosis of infective endocarditis. Clin Infect Dis 2000;30(4):633-638. [http://dx.doi. org/10.1086/313753]

5. Habib G, Derumeaux G, Avierinos JF. Value and limitations of the Duke criteria for the diagnosis of infective endocarditis. J Am Coll Cardiol 1999:33(7):2023-2029.

6. Baggett M, Turbett SE, Schwartzenberg SS, Stone JR. Case records of the Massachusetts General Hospital: Case 5: A 59-year-old man with fever, confusion, thrombocytopenia, rash, 59-year-old man with fever, confusion, thrombocytopenia, rash,
and renal failure. N Engl J Med 2014;370(7):651-660. [http:// dx doi.org/10.1056/NEJMcpc1310004]

7. Sexton DJ, Fowler VG. Clinical manifestations and diagnosis of infective endocarditis. UpToDate 2015. http://www.uptodate. com (accessed 1 July 2015).

8. Hoen B, Duval X. Clinical practice. Infective endocarditis. N Engl J Med 2013;368(15):1425-1433. [http://dx.doi.org/10.1056/ NEJMcp1206782]

9. Brusch JL. Infective Endocarditis: Clinical Presentation. http:// emedicine.medscape.com/article/216650-216650 (accessed 13 August 2015).

10. Yasar KK, Pelivanoglu F, Gursoy S, Sengoz G. Tricuspid endocarditis and septic pulmonary embolism in an intravenous endocarditis and septic pulmonary embolism in an intravenous
drug user with advanced HIV infection. Oman Med J 2011;26(5):365-367.

11. Habib G, Badano L, Tribouilloy C, et al. Recommendations for the practice of echocardiography in infective endocarditis. Eur J Echocardiogr 2010;11(2):202-219. [http://dx.doi.org/10.1093/ ejechocard/jeq004]

12. Mügge A, Daniel WG, Frank G, Lichtlen PR. Echocardiography in infective endocarditis: Reassessment of prognostic implications of vegetation size determined by the transthoracic and the transesophageal approach. J Am Coll Cardiol 1989;14(3):631-638.

13. Rasmussen RV, Høst U, Arpi M, et al. Prevalence of infective endocarditis in patients with Staphylococcus aureus bacteraemia: The value of screening with echocardiography. Eur J Echocardiogr 2011:12(6):414-420. [http://dx.doi.org/10.1093/ ejechocard/jer023]

14. Fournier PE, Thuny F, Richet H, et al. Comprehensive diagnostic Fournier PE, Thuny $\mathrm{F}$, Richet $\mathrm{H}$, et al. Comprehensive diagnostic strategy for blood culture study of 819 new cases. Clin Infect [http://dx.doi.org/10.1086/653675]

5. Oliver S, Whitelaw A, Bamford C. Western Cape Academic Hospitals Antimicrobial Recommendations 2013. Cape Town: National Health Laboratory Service, 2013.

6. Thuny F, Beurtheret S, Mancini J, et al. The timing of surgery influences mortality and morbidity in adults with severe complicated infective endocarditis: A propensity analysis. Eur Heart J 2011;32(16):2027-2033. [http://dx.doi.org/10.1093/ eurhearti/ehp089]

17. Chu V, Cabell CH, Benjamin DK Jr, et al. Early predictors of in-hospital death in infective endocarditis. Circulation 2004;109(14):17451749. [http://dx doiorg/10.1161/01.CIR.0000124719.61827.7F]

8. San Román JA Ĺpez Jilacosta I et al Prognostic statification

8. San Román JA, López J, Vilacosta I, et al. Prognostic stratification of patients with left-sided endocarditis determined at admission. amimed 2006.05.071] 